\title{
Understanding the mode of phytohormones' action in plants
}

\author{
GUO HongWei \\ State Key Laboratory of Protein and Plant Gene Research, College of Life Sciences, Peking University, Beijing 100871, China
}

Received November 24, 2011; accepted December 4, 2011

Citation: Guo H W. Understanding the mode of phytohormones' action in plants. Sci China Life Sci, 2011, 54: 1062-1063, doi: 10.1007/s11427-011-4246-y

Phytohormones are several small molecules that play essential roles throughout plant lifespan. Since in 1880 Charles Darwin and his son Francis Darwin discovered that plant phototropism is regulated by a certain substance which is transported from coleoptile tips, phytohormones have been studied for more than a century. Meanwhile, auxin, gibberellin, cytokinin, abscisic acid, ethylene, jasmonic acid, salicylic acid and brassinosteriod have been identified as eight major hormones regulating responsiveness to various internal and external signals in plants.

During the past decades, forward and reverse genetic methods have been used to dissect the mechanism of phytohormone actions [1]. Plenty of molecular details in the biosynthesis, perception as well as signal transduction pathways of these hormones have been well elucidated, largely based on the research in the model organisms Arabidopsis thaliana and Oryza Sativa (rice). Meanwhile, while new functions of phytohormones in plant growth and development as well as responses to environmental changes have been uncovered, accumulating evidence referring to signal cross-talks among distinct hormones at different levels has been documented. On the other hand, the new sequencing technology and comparative genomics approaches greatly facilitated plant hormone studies in other non-model species. From the investigations into lower plants such as Physcomitrella patens and Selaginella moellendorffii, the evolutionary history of some hormones' actions began to unfold. Thanks to the comparable features and commonality in the action mechanisms of phytohormones, those findings made in the model organisms could be directly utilized to modify hormone responses to generate high yield, more stress tolerant and better quality crops.

email: hongweig@pku.edu.cn
However, despite the significant advances made in this field, a large amount of questions concerning regulation of hormone metabolism, biochemical nature of hormone signal perception and transduction, extensive signal cross-talks and seemly complicated regulatory network, as well as the origin and evolution of diverse hormonal effects on growth, development, defense and adaptation remain to be addressed. More efforts should be taken to understand the hormone signaling in a comprehensive and systematic view. In addition, quantifying the endogenous hormone levels in distinctive plant tissues accurately, sensitively and lively is still a great challenge that would remain an obstacle to the further understanding of hormone metabolism, transport, and action. In an effort aiming to help tackle these problems, several research programs supported by the Chinese funding agencies have been initiated and complemented in the past decade, and significant progress has been achieved. To partly summarize these achievements, a special topic had been organized on the actions of plant hormones in the English version of Chinese Science Bulletin (2010, Vol.55, Number 21). In this Special Topic, a brief description of the program "Molecular Mechanisms of Plant Hormone Actions" recently initiated and funded by the National Natural Science Foundation of China (NSFC) was first introduced and discussed by Li ChuanYou and Li JiaYang [2]. They introduced the research scopes covered in this program, the scientific goals, and the steering committee of the program consisting of seven experts in the fields of botany and chemistry. Following this introduction, three reviews and three research articles in hormone quantification and hormone analysis were also included in this Special Topic [3,4,5-8].

Epigenetic regulation is suggested to participate in hormone actions extensively, as reviewed by Zhu YuXian [3], and various aspects of epigenetic regulation such as DNA 
methylation, chromatin remodeling, histone modification, microRNAs and siRNAs are overviewed and discussed in regulation of hormone-mediated gene expression. Though the current understanding of epigenetic mechanism in phytohormone signaling is generally poor, epigenetic control of stress responses involving ABA biosynthesis, perception and signaling is becoming evident. Scientists also attempt to identify numerous microRNAs, siRNAs and DNA methylation components that play roles in hormone response pathways from emerging sequencing and annotation data.

As mentioned previously, hormone research in crops is demanded for its application potential and for revealing diversity and adaptation of hormone regulation. Ma et al. [4] reviewed ethylene signaling in rice. As a gaseous hormone, ethylene plays essential roles in plant growth, development and response to environmental changes. The linear pathway of ethylene signal transduction has been well studied in the model organism Arabidopsis thaliana [9]. However, the knowledge of its roles and signal transduction in monocotyledon such as rice lags behind. So far, almost each orthologue gene of ethylene signaling component in rice has been cloned and several of them have been demonstrated to function similarly as in Arabidopsis. Nonetheless, additional components have also been unraveled that play a part in myriad adaptive characteristics of rice, such as coleoptile elongation, shoot elongation, adventitious root emergence and aerenchyma development. Furthermore, a few novel signaling components, named as MHZs, have been implicated in ethylene signaling pathway in rice, although their molecular features and relationship with the canonical ETR1-EIN2-EIL pathway remain to be investigated.

In plants, the dynamic metabolism of some phytohormones serves as an essential mechanism in phytohormones' action. Methylation is one of the most common modifications to these small molecules, and surprisingly, all the phytohormone methyltransferases discovered till now are members of the SABATH family. Qu et al. [5] summarized our current knowledge about this family, especially members that function in hormone metabolism. Since it is demonstrated that methylation may affect the activity, volatility and transport of hormones, these methyltransferases must play a role in different hormone actions. Their functions in modulating phytohormone actions according to different external and internal stimulus as well as their substrate specificity from the structural aspect of view were also discussed in this review. In addition, de-methylation and its function in plant development were also discussed.

In plant hormone biology, accurate and sensitive quantification of endogenous levels of hormones and their derivatives in various plant tissues is still a great challenge. Concerning this difficulty, in this Special Topic, three articles are dedicated to the new advances in hormone analysis and measurement. The method of identifying and quantifying two isomers of brassinosteroids, 28-homobrassinolide (28-h-BL) and 28-epihomobrassinolide (28-eh-BL) using tandem mass spectrometry with the electrospray ionization (ESI) had been described by Huo et al. [6]. Liao et al. [7] reported a rapid procedure, by which they increased the sensitivity and accuracy of measuring endogenous methyl jasmonate based on the molecular electrochemical characteristic, while Liu et al. [8] utilized liquid chromatography tandem mass spectrometry, which led to increased sensitivity in the quantification of both jasmonic acid and methyl jasmonate. Since different isomers of phytohormones could have distinct biological functions [5], accurate determination of the various forms of endogenous phytohormones would no doubt advance the study of their actions.

Apparently, these reviews and articles in this Special Topic had only covered a small portion of recent progress in phytohormone studies. However, it is evident that achievements in this field in China are encouraging. The effects of phytohormone on plant growth and development have been exploited in agriculture for dozens of years. However, several practical issues still remain to be solved, such as the usage efficacy, side-effects, as well as environmental safety. Further investigation on the determination, economical production, and action mode of phytohormones would greatly help address these issues. As the population grows, agriculture is an emergent issue in China. To solve it, NSFC has carried out a series of research programs, and substantial progress has been made to date. It shows that the supportive policy can bring us more confidence and probably more solutions for the problems related to the food safety of the country.

The author thanks Jiang ZiQiang and Wan Wen in Guo lab for assistance in writing this essay.

1 Alonso J M, Ecker J R. Moving forward in reverse: Genetic technologies to enable genome-wide phenomic screens in Arabidopsis. Nat Rev Genet, 2006, 7: 524-536

2 Li C Y, Li J Y. Toward understanding the molecular mechanisms governing plant hormone actions: A brief introduction to the Major Research Program "Molecular mechanism of plant hormone actions" funded by the National Natural Science Foundation of China (NSFC). Chin Sci Bull, 2010, 55: 2197

3 Zhu Y X. The epigenetic involvement in plant hormone signaling. Chin Sci Bull, 2010, 55: 2198-2203

4 Ma B, Chen S Y, Zhang J S. Ethylene signaling in rice. Chin Sci Bull, 2010, 55: 2204-2210

5 Qu L J, Li S, Xing S F. Methylation of phytohormones by the SABATH methyltransferases. Chin Sci Bull, 2010, 55: 2211-2218

6 Huo F F, Bai Y, Liu H W. Fragmentation study of two brassinolides by ion trap tandem mass spectrometry. Chin Sci Bull, 2010, 55: 2219-2224

7 Liao Y Q, Wang F, Chen Z L. Electrochemical behavior and the determination of methyl jasmonate. Chin Sci Bull, 2010, 55: 2225-2230

8 Liu X, Yang Y L, Lin W H, et al. Determination of both jasmonic acid and methyl jasmonate in plant samples by liquid chromatography tandem mass spectrometry. Chin Sci Bull, 2010, 55: 2231-2235

9 Zhao Q, Guo H. Paradigms and paradox in the ethylene signaling pathway and interaction network. Mol Plant, 2011, 4: 626-634

Open Access This article is distributed under the terms of the Creative Commons Attribution License which permits any use, distribution, and reproduction in any medium, provided the original author(s) and source are credited. 